\title{
The disease-specific proteins in striatum of the mouse models of Parkinson's disease induced by rotenone and MPTP
}

\author{
Changqing Zhou ${ }^{\mathrm{a}, *}$, Shanshan $\mathrm{Li}^{\mathrm{b}}$, Min Wang ${ }^{\mathrm{c}}$, Zhongmei Chen ${ }^{\mathrm{d}}$, Guoguang Peng ${ }^{\mathrm{d}}$ \\ a Department of Neurology, The Bishan Hospital, Bishan, Chongqing 402760 China \\ b Department of Neurology, Bin Zhou People's Hospital, Binzhou, Shandong 256600 China \\ c Department of Gynaecology and Obstetrics, The Bishan Hospital, Bishan, Chongqing 402760 China \\ d Department of Neurology, The First Affiliated Hospital of Chongqing Medical University, Yuzhong, \\ Chongqing 400016 China
}

*Corresponding author, e-mail: changqing_zhou@163.com

\begin{abstract}
Parkinson's disease (PD) is the second most common neurodegenerative disease. To explore the diseasespecific proteins (DSPs) of PD, two mouse models were established with rotenone and 1-methyl-4-phenyl-1, 2, 3, 6-tetrahydropyridine (MPTP). A strategy of two-dimensional gel electrophoresis (2-DE) in combination with matrixassisted laser desorption ionization time-of-flight mass spectrometry (MALDI-TOF-MS) was used to identify the common differentially expressed proteins in striatum. There were significant differences in behavioral evaluation and the numbers of tyrosine hydroxylase (TH) positive neurons between the rotenone group and control group 1 and between the MPTP group and control group $2(p<0.01)$. Maps of 2-DE were analyzed with PDQuest 8.0 software and 21 differentially expressed proteins were found between rotenone group and control group 1; 27 were found between MPTP group and control group 2. Six common differentially expressed proteins were found in the two mouse models of PD and 3 of them were ultimately identified successfully with MALDI-TOF-MS and database analysis: FEM1B, Pyridoxal kinase and $\alpha$-SNAP. All the 3 common differentially expressed proteins were down-regulated in rotenone group and MPTP group compared with control group 1 and control group 2, respectively. These proteins are primarily associated with apoptosis, degradation of proteins, synthesis and transportation of neurotransmitters and they may be the DSPs of PD.
\end{abstract}

KEYWORDS: biomarkers, proteomics, striatum, two-dimensional gel electrophoresis, time-of-flight mass spectrometry

\section{INTRODUCTION}

Parkinson's disease (PD) is the second most common neurodegenerative disease with a prevalence of $1.7 \%$ in old people aged more than 65 [1]. The clinical symptoms of PD are characterized by resting tremors, rigidity, bradykinesia and postural instability [2]. It is widely accepted that those symptoms of PD are the consequence of the loss of dopaminergic neurons in substantia nigra (SN) and the depletion of dopamine in striatum [3]. The striatum is the main input station of basal ganglia system. It consists of the nigrostriatal pathway with $\mathrm{SN}$ and is most affected in early PD [4].

The etiology and pathogenesis of PD are very intractable and there is no sensitive or specific biomarker and imaging change for diagnosis so far [5]. Therefore, PD is mainly diagnosed based on clinical symptoms. Many clinical studies on proteomics utilized cerebrospinal fluid or blood of PD patients to find the disease-specific proteins (DSPs) of PD for diagnosis while many animal model studies utilized the substantia nigra of a single animal model of PD to study the DSPs $[6,7]$. But they all failed to find out the DSPs of PD for diagnosis [8]. The pathology changes of chronic animal models induced by rotenone and MPTP resemble the changes of PD patients [9]. However, there is few studies focusing on striatum and utilizing chronic multiple animal models to study the DSPs of PD. In this work, a proteomic strategy was applied to explore the common differentially expressed proteins as the DSPs of PD using the samples of striatum from two chronic mouse models induced by rotenone and MPTP. 


\section{MATERIALS AND METHODS}

\section{Materials}

Forty healthy C57BL/ 6 male mice, aged 8-12 weeks, weighing 18-22 g, of clean grade, were provided by the Experimental Animal Center of Chongqing Medical University, with the license No. SYXK (yu) 20140001. Mice were housed 3-4 mice per cage with free access to food and water. The room was maintained at $20 \pm 2{ }^{\circ} \mathrm{C}$ on a $12 \mathrm{~h}$ light/ dark cycle. The experiment protocol complied with the Guidance Suggestions for the Care and Use of Laboratory Animals, issued in 2006 by the Ministry of Science and Technology of China [10]. Rotenone, MPTP were purchased from Sigma (St. Louis, MO, USA). Clean-up kit, solid phase $\mathrm{pH}$ gradient strip (pH 3-10NL, $17 \mathrm{~cm}$ ) and Bio-Lyte were purchased from Bio-Rad (California, USA). Anti-rabbit tyrosine hydroxylase (TH) multi-clone antibody was purchased from Boster (Hubei, China). The firstdimension isoelectric focusing unit and elements, vertical electrophoresis system and elements and PDQuest 8.0 image analysis software were from BioRad. Voyager DE-PROMALDI-TOF-MS mass spectroscopy analyzer was from ABI (California, USA).

\section{Methods}

\section{Establishment of rotenone and MPTP models in mice}

After a week of adaptation to the environment, mice in the rotenone group received subcutaneous injections of rotenone $(1 \mathrm{mg} / \mathrm{kg})$ once a day for 40 successive days and mice in its control group 1 received the same volume of solvent injections according to the paradigm of rotenone group; mice in the MPTP group received subcutaneous injections of MPTP (25 mg/kg) $30 \mathrm{~min}$ after the peritoneal injection of probenecid $(250 \mathrm{mg} / \mathrm{kg}$ ) twice a week for 5 successive weeks and mice in its control group 2 received the same volume of solvent injections after the peritoneal injection of probenecid according to the paradigm of MPTP group.

\section{Behavioral evaluation and tyrosine hydroxylase- immunohistochemical staining}

General activities were observed everyday and open-field test was performed at the 2 nd day of last injection. The modified method of Kawai [11] was applied. The apparatus consisted of a box (30-cm long, 30-cm wide and 15-cm deep), the bottom of which was divided into 25 areas. A mouse was placed in the center of field, and its ambulation and rearing were recorded for 5 successive min and averaged as parameters of locomotor activity.

The expression of $\mathrm{TH}$ in $\mathrm{SN}$ was detected by immunohistochemical staining using a conventional avidin-biotin-immunoperoxidase technique. Briefly, paraffin sections were dewaxed in xylene, hydrated in decreasing percentage of alcohol, then washed for $5 \mathrm{~min}$ in $0.01 \mathrm{M}$ phosphate-buffered saline and treated with $0.3 \%$ hydrogen peroxide. Then the sections were washed 3 times for $5 \mathrm{~min}$ in $0.01 \mathrm{M}$ PBS, followed by $30 \mathrm{~min}$ of preincubation with $10 \%$ normal calf serum. The brain sections were then incubated with anti-TH antibody (1:150) overnight at $4{ }^{\circ} \mathrm{C}$. After a $15 \mathrm{~min}$ rinse in changes of PBS, the sections were incubated with biotinylated secondary antibody for $20 \mathrm{~min}$ and then with avidin-biotin peroxidase complex for $20 \mathrm{~min}$ at room temperature. Immunoreactions were visualized using 0.05\% diaminobenzidine after the sections were washed with PBS. Then, dehydrated in increasing percentage of alcohols, till 70\% alcohol, differentiated in $90 \%$ alcohol, cleared in xylene and finally mounted. The TH-positive neurons were observed under light microscope (Olympus, Tokyo, Japan).

\section{Protein extraction and two-dimensional gel elec- trophoresis}

Mice were perfused with ice saline after anesthetization with chloral hydrate rapidly. Then they were sacrificed by cervical dislocation. Striatums were dissected referring to the atlas of mouse brain [12] and the method of Thomas [13], frozen with liquid nitrogen rapidly and stored at $-80^{\circ} \mathrm{C}$ until use. The frozen brain tissues were homogenized with sonication (10 watt for $4 \mathrm{~s}$ and $6 \mathrm{~s}$ interval) for $3 \mathrm{~min}$ in buffer consisting of $7 \mathrm{M}$ urea, $2 \mathrm{M}$ thiourea, 4\% CHAPS, $1 \mathrm{mM}$ PMSF, $40 \mathrm{mM}$ Tris-base and $65 \mathrm{mM}$ DTT. The tissue was further treated with $20 \mathrm{~g} / 1$ DNase I and $5 \mathrm{~g} / 1$ RNase A at $4{ }^{\circ} \mathrm{C}$ for $30 \mathrm{~min}$, and centrifuged at $40000 \mathrm{~g}$ at $4{ }^{\circ} \mathrm{C}$ for $30 \mathrm{~min}$. Supernatants were collected avoiding lipids. Protein extracted from striatums was treated with Clean-up kit, following the manufacturer's protocols. The protein concentration of the final extract solution was determined using the modified Bradford method.

Immobiline IPG dry strip gels were hydrated with buffer consisting of $7 \mathrm{M}$ urea, $2 \mathrm{M}$ thiourea, 4\% CHAPS, 0.2\% Bio-Lyte and $65 \mathrm{mM}$ DTT in a total volume of $400 \mu \mathrm{l}$ at $17^{\circ} \mathrm{C}$ for $12 \mathrm{~h}$. And the strips were loaded with $180 \mu \mathrm{g}$ of protein sample at the same time of hydration. IEF was performed at $17^{\circ} \mathrm{C}$ 
using the first-dimension isoelectric focusing units following the manufacturer's protocols. For IEF, the voltage was linearly increased from 250 to $10000 \mathrm{~V}$ for $8.5 \mathrm{~h}$ for sample entry followed by a constant $10000 \mathrm{~V}$, with the focusing complete after $60 \mathrm{kVh}$. Prior to the second dimension, strips were incubated for $15 \mathrm{~min}$ in equilibration buffer $(0.375 \mathrm{M}$ Tris$\mathrm{HCl}, \mathrm{pH} 6.8$ containing $6 \mathrm{M}$ urea, $2 \%$ SDS, and $20 \%$ glycerol), first with $2 \%$ DTT and second with $2.5 \%$ TAA. The equilibrated strips were inserted onto SDSPAGE gels (12\%). SDS-PAGE was performed using the vertical electrophoresis system and elements, following the manufacturer's protocols. The gels were run at the current of $5 \mathrm{~mA}$ per gel for $30 \mathrm{~min}$, $30 \mathrm{~mA}$ per gel for $7 \mathrm{~h}$ at $17^{\circ} \mathrm{C}$, then SDS-PAGE gels were silver-stained.

\section{Image analysis}

The silver-stained gels were scanned. In order to minimize the variations that arose from sample loading, run-to-run procedure and staining variability, quantitative analysis of the digitized maps were analyzed with PDQuest 8.0 software according to the protocols provided by the manufacturer. Spots were detected automatically and matched on the 3 images, followed by manual editing of spots and spot alignment by an experienced user to improve detection and eliminate artifacts. Differentially expressed protein spots were selected statistically for the significant expression of variation that deviated over about 1.5 folds in their expression levels compared with the control group.

\section{Matrix-assisted laser desorption ionization time-of-flight mass spectrometry (MALDI-TOF- MS) and database analyses}

The differentially expressed protein spots were excised, decolorized, dried, digested with sequence grade porcine trypsin, and added to matrix, then spotted onto the target plate. Protein analysis was performed using an Voyager DE-PROMALDI-TOFMS. (Analysis parameters were the same as those described by Chen [14].) Peptide mass fingerprints were then retrieved in Mascot Swiss-Prot database to identify the protein spots (www.matrixscience. com). The parameters were set as follows: database was SwissProt; taxonomy was Mus.; enzyme was Trypsin; missed cleavage was 1 ; fixed modification was Carbamidomethyl (C); variable modifications were Acetyl (Protein N-term) and Oxidation (M); Peptide tol.was +1.2 Da; Mass values was $\mathrm{MH}+$; Monoisotopic was Average. The basic requirement for identification was that the expectation value
Table 1 Behavioral evaluation of the 4 groups.

\begin{tabular}{lcc}
\hline Group & Ambulation score & Rearing score \\
\hline Rotenone & $59.22 \pm 4.42^{\mathrm{a}}$ & $19.60 \pm 1.67^{\mathrm{a}}$ \\
Control 1 & $92.92 \pm 7.15$ & $27.58 \pm 3.59$ \\
MPTP & $60.18 \pm 3.08^{\mathrm{b}}$ & $19.98 \pm 1.45^{\mathrm{b}}$ \\
Control 2 & $94.62 \pm 6.38$ & $26.90 \pm 2.39$ \\
\hline
\end{tabular}

Data are expressed as mean $\pm \mathrm{SD}$ and there are 10 mice in each group. a, $p<0.01$ versus control group 1 ; b, $p<0.01$ versus control group 2 .

(chance of misidentification) was less than 0.05 and the coverage was more than $20 \%$ as described by Wang [15].

\section{Statistical analysis}

The data were expressed as the mean \pm SD. A statistical analysis was performed using SPSS 20.0 software (Chicago, IL, USA) with an analysis of $t$-test. In all analyses, statistical significance was defined as $p<0.05$.

\section{RESULTS}

\section{Subcutaneous injection of rotenone and MPTP produced behavioral deficits in mice}

Mice in rotenone group and MPTP group showed piloerection, tremor, bradycardia and lower reactive to stimulus after injection. Those symptoms were more obvious and lasted long, but they all disappeared on the next day. However, no behavioral changes were observed in control group 1 and control group 2. The scores of ambulation and rearing in rotenone group and MPTP group were reduced significantly in the open-field test compared with control group 1 and control group 2, respectively $(p<0.01)$ (Table 1).

\section{Rotenone and MPTP reduced the TH-positive neurons in $\mathrm{SN}$}

Tyrosine hydroxylase-immunohistochemical staining was performed to validate the establishment of animal models. The results showed that the injection of rotenone and MPTP reduced the expression of tyrosine hydroxylase in SN (Fig. 1A). In rotenone group and MPTP group, the numbers of TH-positive neurons had significantly decreased in the SN compared with control group 1 and control group 2 , respectively ( $p<0.01)$ (Fig. 1B). 


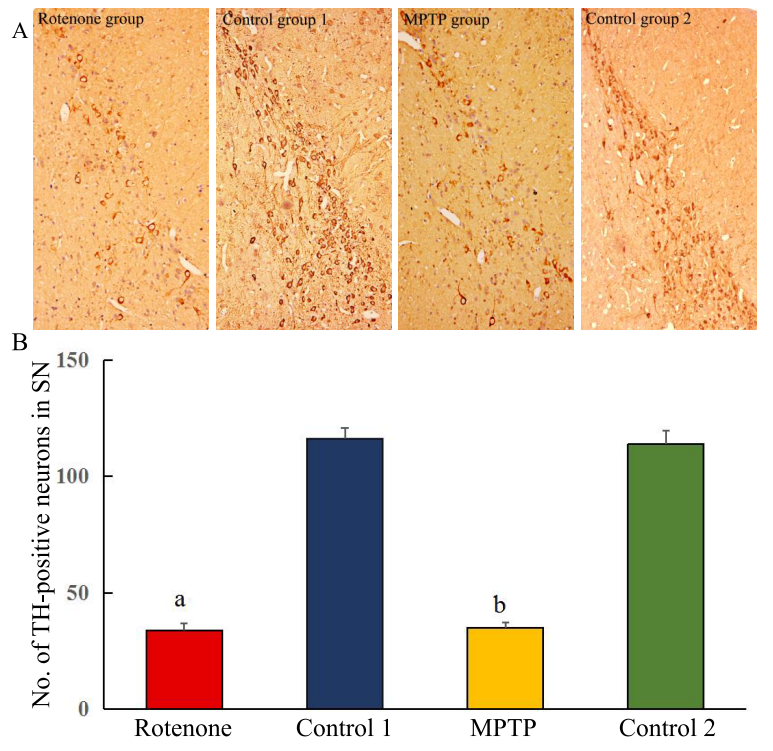

Fig. 1 The expression of TH in SN by immunohistochemical staining. (A) Rotenone group, control group 1, MPTP group and control group 2, respectively $(\times 100)$. (B) Compared with control group 1 and control group 2, the numbers of TH-positive neurons were significantly decreased in the rotenone group and MPTP group. The numbers are expressed as mean $\pm \mathrm{SD}$; a, $p<0.01$ versus control group 1 ; b, $p<0.01$ versus control group 2 .

\section{Two-dimensional gel electrophoresis protein maps and grouping}

Forty mice were randomly assigned to 4 groups $(n=10)$ : rotenone group and its control group 1 and MPTP group and its control group 2. Mice in the rotenone group and control group 1 received subcutaneous injections of rotenone and the same volume of solvent, respectively; mice in the MPTP group and control group 2 received subcutaneous injections of MPTP and the same volume of solvent, respectively, $30 \mathrm{~min}$ after the injection of probenecid. Two-dimensional gel electrophoresis (2-DE) was performed with the protein extracted from striatum to obtain the protein maps of each group. Fig. 2 shows the 2-DE protein maps of the four groups. The digitized maps were analyzed with PDQuest 8.0 software according to the protocols and up to 1220 protein spots were detected in each map. The differentially expressed proteins were selected for further analysis when $p<0.05$ and the average fold change was $>1.5$ or $<-1.5$. A total of 21 differentially expressed proteins were found between the rotenone group and control group 1; in those proteins, 9 were up-regulated and
Table 2 Identification of the 3 common differentially expressed protein spots.

\begin{tabular}{lllccc}
\hline Spot & NCBI & Protein & $\begin{array}{c}\text { MW/IP } \\
\text { (kDa) }\end{array}$ & $\begin{array}{c}\text { Matched } \\
\text { peptide }\end{array}$ & $\begin{array}{c}\text { Mascot } \\
\text { score }\end{array}$ \\
\hline 1 & Q9Z2G0 & FEM1B & $71.2 / 6.1$ & $27(39 \%)$ & 63 \\
2 & Q8K138 & Pyridoxal kinase & $35.3 / 5.9$ & $19(50 \%)$ & 70 \\
3 & Q9DB05 & $\alpha$-SNAP & $33.6 / 5.3$ & $27(88 \%)$ & 98 \\
\hline
\end{tabular}

NCBI, National Center for Biotechnology Information; MW, molecular weight; IP, isoelectric point.

12 were down-regulated. While 27 differentially expressed proteins were found between the MPTP group and control group 2; in those proteins, 11 were up-regulated and 16 were down-regulated. Six common differentially expressed proteins were ultimately found in those 2 groups; of the 6 proteins, 2 were up-regulated and 4 were down-regulated.

\section{MALDI-TOF-MS and Database analyses}

The 6 common differentially expressed proteins were cut from the gels, then digested and analysed by MALDI-TOF-MS. The peptide mass fingerprints were retrieved in the Mascot Swiss-Prot database to identify the protein spots. Three proteins were ultimately identified in the database successfully: FEM1B, Pyridoxal kinase and $\alpha$-SNAP (Table 2). All the 3 common differentially expressed proteins were down-regulated in rotenone group and MPTP group compared with control group 1 and control group 2, respectively (Fig. 2). The peptide mass fingerprinting spectra and Mascot score histograms of the 3 common differentially expressed protein spots were shown in Fig. 3.

\section{DISCUSSION}

Proteomics is a further study of genomics. It does not only study proteins which are the direct function performers of life, but it is also more consistent with the views of systemic biology. Proteomics is widely applied in various research fields of bioscience, especially in the DSP and target studies of serious diseases in human $[16,17]$. The proteomic studies of PD usually focus on single acute and transgene animal models. However, there are few studies focusing on chronic multiple animal models of PD which better mimic the pathology of PD $[18,19]$. Rotenone and MPTP are the common neurotoxic substances in the animal model studies of PD and the pathology changes of chronic mouse models induced by rotenone and MPTP resemble the changes of PD patients [9]. Therefore it is possible to find out the DSPs of PD through comparing the differentially 

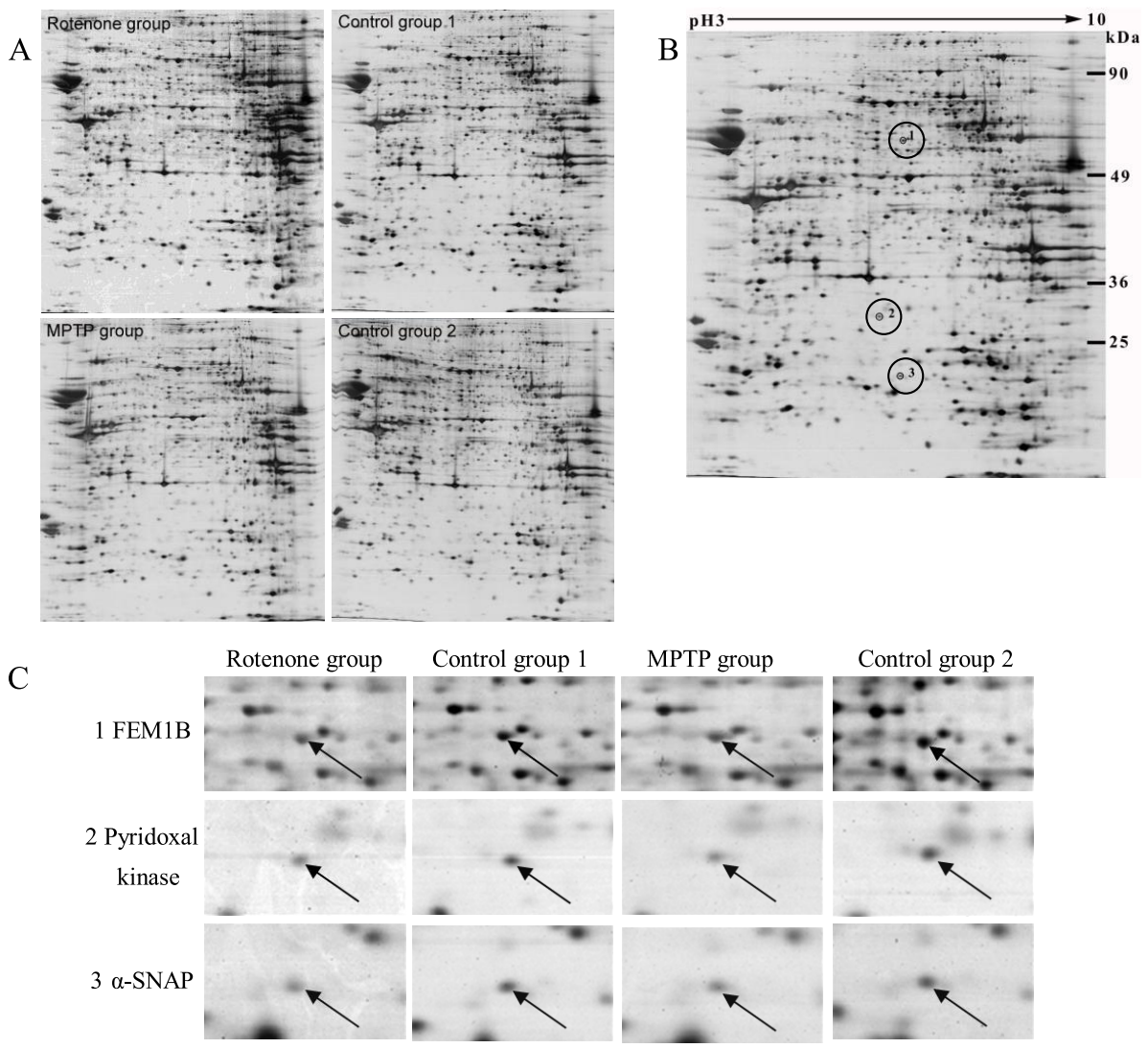

Fig. 2 Protein maps with differential expression. (A) Striatum tissue protein maps of the four groups over pH 3-10 range. (B) The locations of the 3 common differentially expressed protein spots in the 2-DE map. (C) The common differentially expressed protein spots in the 4 groups. Arrowheads represent protein spots that showed significantly different changes between experiment and control groups.

expressed proteins in those two animal models. This study has broken through the traditional proteomics research pattern to explore the common differentially expressed proteins of striatum in two mouse models of PD induced by rotenone and MPTP. Three of the common differentially expressed proteins were ultimately identified successfully: FEM1B, Pyridoxal kinase and $\alpha$-SNAP. All of the 3 proteins were down-regulated in rotenone group and MPTP group. They are primarily associated with apoptosis, degradation of proteins, synthesis and transportation of neurotransmitters and may be the DSPs of PD.

Protein fem-1 homolog B (FEM1B) belongs to the fem-1 family and locates in cytoplasm and nucleus. As the substrate recognition subunit, it composes of E3 ubiquitin-protein ligase complex with CUL2, RBX1, TCEB1 and TCEB2 [20]. E3 ubiquitinprotein ligase plays an important role in the ubiquitin proteasome system. Studies showed that the mutation of E3 ubiquitin-protein ligase might cause the deposition of unfolded or misfolded proteins, such as $\alpha$-synuclein, in parkinson's disease and other neurodegenerative disorders [21]. Furthermore, Fem $1 \mathrm{~b}$ is a proapoptotic protein that induces apoptosis when expression is increased in cancer cells including colon cancer, breast cancer, cervical cancer and neuroblastoma [22]. Upstream, Fem1b binds to apoptosis-inducing death receptors Fas and tumor necrosis factor receptor-1 (TNFR1) in the cell surface [23]. Down-stream, Fem1b binds to apoptotic protease activating factor-1 (Apaf-1), a core component of the apopto-some which is a major regulator of caspase activation and initiation of apoptosis. Bcl-XL, a dominant negative Fas-associated death domain or a dominant-negative caspase-9, can inhibit Fem1b-induced apoptosis [23].

The present results demonstrated that the protein levels of FEM1B were down-regulated in both rotenone group and MPTP group compared with 

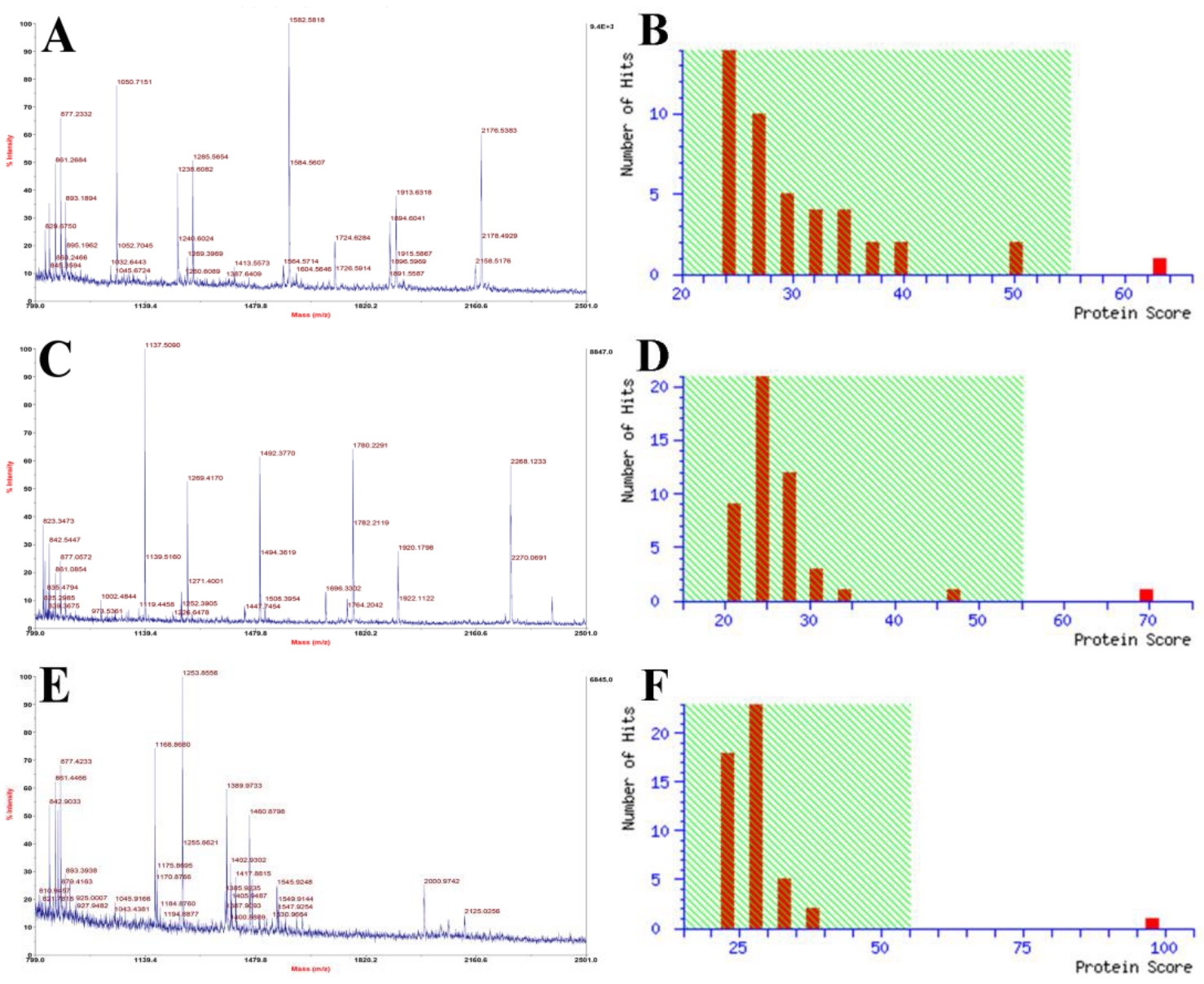

Fig. 3 The peptide mass fingerprinting spectra and Mascot score histograms of the 3 common differentially expressed protein spots. (A,C,E) represent the peptide mass fingerprinting spectra of FEM1B, Pyridoxal kinase and $\alpha$-SNAP. Y-axis represents protein signal peak intensity; $\mathrm{X}$-axis represents mass charge ratio $(\mathrm{m} / \mathrm{z})$ in the peptide mass fingerprinting spectra. (B,D,F) represent the Mascot score histograms of FEM1B, Pyridoxal kinase and $\alpha$-SNAP. Y-axis represents number of hits; $\mathrm{X}$-axis represents protein score in the Mascot score histograms.

the control groups, respectively. It is well known that the dysfunction of Ubiquitin-proteasome system and apoptosis are involved in development of $\mathrm{PD}$. And FEM1B is the substrate recognition subunit of E3 ubiquitin-protein ligase complex and acts as the death receptor-associated protein that mediates apoptosis. Therefore, those findings suggest that FEM1B may be the DSP of PD.

Pyridoxal kinase belongs to the vitamin $\mathrm{B} 6 \mathrm{ki}$ nase family and distributes in the cytoplasm. It is involved in the metabolism of vitamin B6. Pyridoxal kinase synthesizes $5^{\prime}$-pyridoxal phosphate by transferring phosphoryl group in the presence of cofactors zinc and magnesium [24]. 5'-pyridoxal phosphate is the only active form of vitamin B6 and as the cofactor is involved in the metabolism of amino acids, fat and glycogen. Pyridoxal kinase was involved in the neural degeneration in Huntington's disease and it was oxidized in the brains of Huntington's disease patients and mouse models [25]. Further study showed that the oxidation of pyridoxal kinase resulted in the reduction of $5^{\prime}$ pyridoxal phosphate synthesized and affected the synthesis of GABA and dopamine finally. Study had confirmed vitamin B6 could alleviate the occurrence of PD $[26,27]$ and the genetic mutation of pyridoxal kinase was associated with PD in the study of single cell expression profiling [28]. A multicenter clinical study in Germany, Italy and England analyzed the entire gene expression profile of SN and the differently expressed genes labeled SNPs in PD patients and found that the mutation of 
PDXK gene encoding pyridoxal kinase increased the occurrence of PD $[29,30]$. The mechanism may be related with the reduction of $5^{\prime}$-pyridoxal phosphate synthesized due to the mutation of pyridoxal kinase, then $5^{\prime}$-pyridoxal phosphate, as the cofactor of dopa decarboxylase, would reduce the transformation to dopamine.

In the present study, we found pyridoxal kinase was down-regulated in both rotenone group and MPTP group compared with the control groups, respectively. In a previous proteomics study of substantia nigra, pyridoxal kinase which plays an important role in the neural degeneration was also found to be down-regulated in mice models of PD compared with the control groups [31], which is in accordance with the present study to some extent. Therefore, pyridoxal kinase may be the DSP of PD.

Alpha-soluble N-ethylmaleimide-sensitive factor (NSF) attachment protein ( $\alpha$-SNAP) belongs to the SNP family and locates in the cell membrane, especially in synaptic vesicle and presynaptic plasma membranes. It is involved in the vesicular transport between the endoplasmic reticulum and the golgi apparatus and plays an essential role in membrane fusion by bridging cis SNARE complexes to the NSF [32]. $\alpha$-SNAP stimulates NSF to release itself and recycles individual SNARE to reengage in the trans arrays for subsequent fusion reaction [33]. Other study showed that the activity of NSF ATPase stimulated by $\alpha$-SNAP was required for SNARE complex disassembly and exocytosis [34]. Furthermore, $\alpha$-SNAP was found to interact with agnoprotein to modulate exocytosis in the study of human polyomavirus BK [35]. Alteration of $\alpha$-SNAP may be related to central nervous system diseases [36]. Reduced expression of $\alpha$ SNAP was reported in Down's syndrome, temporal lobe epilepsy (TLE) and Creutzfeldt-Jakob disease (CJD); however, increased $\alpha$-SNAP expression was reported in Huntington's disease. Previous study showed that the function defect of vesicular might change the concentration of dopamine in PC12 cells [37]. $\alpha$-SNAP phosphorylation decreases its ability to bind the SNARE complex by one order of magnitude [38]. The metabolites and side products of excess dopamine in cytosolic could cause neurodegeneration by triggering oxidative stress pathways in PD [39].

The present results demonstrate the protein levels of $\alpha$-SNAP were down-regulated in both rotenone group and MPTP group compared with the control groups, respectively. Previous studies showed $\alpha$-SNAP was involved in the vesicu- lar transport and the down-regulation of $\alpha$-SNAP might induce the transport disorder of dopamine in dopaminergic neurons in PD. Furthermore, a proteomics study in substantia nigra found the reduced expression of NSF in MPTP-treated mice [40]. $\alpha$-SNAP, which was down-regulated in the present study, can stimulate NSF to release itself. That is to say the reduction of $\alpha$-SNAP may cause the reduction of NSF. Therefor, the reduction of NSF in $\mathrm{SN}$ in previous study is in accordance with the present study. These findings suggest that $\alpha$-SNAP may be the DSP of PD.

In short, the present study utilized two chronic mouse models of PD induced by rotenone and MPTP to study the proteomics changes of striatum. Three common proteins, FEM1B, Pyridoxal kinase and $\alpha$-SNAP, were screened out from the two groups of differentially expressed proteins as the DSPs of PD. They were reported for the first time in the proteomics study of striatum. Their specific mechanisms in the occurrence of PD are needed in further study, but the changes in protein level should provide valuable clues for further study of the biomarkers and new therapy targets for PD.

Acknowledgements: This work was supported by a research grant from the National Natural Science Foundation of China (NO. 30370499). We are very grateful to the colleagues in the Key Laboratory of Neurology of First Affiliated Hospital of Chongqing Medical University.

\section{REFERENCES}

1. Zhang ZX, Roman GC, Hong Z, Wu CB, Qu QM, Huang JB, Zhou B, Geng ZP, et al (2005) Parkinson's disease in China: prevalence in Beijing, Xian, and Shanghai. Lancet 365, 595-597.

2. Wang Y, Li Y, Zhang X, Xie A (2018) Apathy following bilateral deep brain stimulation of subthalamic nucleus in Parkinson's disease: a meta-analysis. Parkinsons Dis 2018, ID 9756468.

3. Stoker TB, Barker RA (2018) Regenerative therapies for Parkinson's disease: an update. BioDrugs 32, 357-366.

4. Caminiti SP, Presotto L, Baroncini D, Garibotto V, Moresco RM, Gianolli L, Volonté MA, Antonini A, et al (2017) Axonal damage and loss of connectivity in nigrostriatal and mesolimbic dopamine pathways in early Parkinson's disease. Neuroimage Clin 14, 734-740.

5. Miller DB, O'Callaghan JP (2015) Biomarkers of Parkinson's disease: present and future. Metabolism 64, 40-46.

6. Sowell RA, Owen JB, Butterfield DA (2009) Proteomics in animal models of Alzheimer's and Parkinson's diseases. Ageing Res Rev 8, 1-17. 
7. Henchcliffe C, Dodel R, Beal MF (2011) Biomarkers of Parkinson's disease and Dementia with Lewy bodies. Prog Neurobiol 95, 601-613.

8. Blandini F, Armentero MT (2012) Animal models of Parkinson's disease. FEBS J 279, 1156-1166.

9. Jincheng Wan, Yuping Zhang, Hanchun Long, Furong Xu, Guoguang Peng (2010) Effect of rotenone on $\alpha$-synuclein expression in substantia nigra of mouse model of Parkinson's disease. Med $J$ Chin PLA 35, 667-670.

10. Ministry of Science and Technology (2006) Guidance Suggestions for the Care and Use of Laboratory Animals, China.

11. Kawai H, Makino Y, Hirobe M, Ohta S (1998) Novel endogenous 1, 2, 3, 4- tetrahydroisoquinoline derivatives: uptake by dopamine transporter and activity to induce parkinsonism. $J$ Neurochem 70, 745-751.

12. Xiong B, Li A, Lou Y, Chen S, Long B, Peng J, Yang Z, $\mathrm{Xu}$ T, et al (2017) Precise cerebral vascular atlas in stereotaxic coordinates of whole mouse brain. Front Neuroanat 19, ID 128.

13. Thomas G, Heffner, John A (1980) A rapid method for the regional dissection of the rat brain. Pharmacol Biochem Behav 13, 453-456.

14. Ying C, Gang Y, Wenbin T, Peng G (2010) Cerebrospinal fluid diagnostic markers for twodimensional electrophoresis-mass spectrometry in Parkinson's disease patients. Neural Regen Res 5, 890-894.

15. Ying Wang, Zhong Dong, Hongyan Fan (2011) Identification of differentially expressed proteins in $\mathrm{SH}-$ SY5Y cells treated with resveratrol. Neural Regen Res 6, 1612-1617.

16. Vohra A, Asnani A (2018) Biomarker discovery in cardio-oncology. Curr Cardiol Rep 20, ID 52.

17. Dahabiyeh LA (2018) The discovery of protein biomarkers in pre-eclampsia: the promising role of mass spectrometry. Biomarkers 23, 609-621.

18. Jagota A, Mattam U (2017) Daily chronomics of proteomic profile in aging and rotenone-induced Parkinson's diseasemodel in male Wistar rat and its modulation by melatonin. Biogerontology 18, 615-630.

19. Kasap M, Akpinar G, Kanli A (2017) Proteomic studies associated with Parkinson's disease. Expert Rev Proteomics 14, 193-209.

20. Kamura T, Maenaka K, Kotoshiba S, Matsumoto M, Kohda D, Conaway RC, Conaway JW, Nakayama K (2004) VHL-box and SOCS-box domains determine binding specificity for Cul2-Rbx1 and Cul5Rbx2 modules of ubiquitin ligases. Genes Dev 18, 3055-3065.

21. Kumar P, Pradhan K, Karunya R, Ambasta RK, Querfurth HW (2012) Cross-functional E3 ligases Parkin and C-terminus Hsp70-interacting protein in neurodegenerative disorders. $J$ Neurochem 120, 350-370.

22. Subauste MC, Sansom OJ, Porecha N, Raich N, Du
L, Maher JF (2010) Fem1b, a proapoptotic protein, mediates proteasome inhibitor-induced apoptosis of human colon cancer cells. Mol Carcinog 49, 105-113.

23. Chan SL, Tan KO, Zhang L, Yee KS, Ronca F, Chan MY, Yu VC (1999) F1Aalpha, a death receptor-binding protein homologous to the Caenorhabditis elegans sex-determining protein, FEM-1, is a caspase substrate that mediates apoptosis. J Biol Chem 274, 32461-32468.

24. Di Salvo ML, Safo MK, Contestabile R (2012) Biomedical aspects of pyridoxal 5'-phosphate availability. Front Biosci 4, 897-913.

25. Shen L (2015) Associations between B vitamins and Parkinson's Disease. Nutrients 7, 7197-7208.

26. Tien LT, Lee YJ, Pang Y, Lu S, Lee JW, Tseng $\mathrm{CH}$, Bhatt AJ, Savich RD, et al (2017) Neuroprotective effects of intranasal IGF-1 against neonatal lipopolysaccharide-induced neurobehavioral deficits and neuronal inflammation in the substantia nigra and locus coeruleus of juvenile rats. Dev Neurosci 39, 443-459.

27. Loens S, Chorbadzhieva E, Kleimann A, Dressler D, Schrader C (2017) Effects of levodopa/carbidopa intestinal gel versus oral levodopa/carbidopa on B vitamin levels and neuropathy. Brain Behav 7, e00698.

28. Elstner M, Morris CM, Heim K, Lichtner P, Bender A, Mehta D, Schulte C, Sharma M, et al (2009) Single cell expression profiling of dopaminergic neurons combined with association analysis identifies pyridoxal kinase as Parkinson's disease gene. Ann Neurol 66, 792-798.

29. Sorolla MA, Rodríguez-Colman MJ, Tamarit J, Ortega Z, Lucas JJ, Ferrer I, Ros J, Cabiscol E (2010) Protein oxidation in Huntington disease affects energy production and vitamin B6 metabolism. Free Radic Biol Med 49, 612-621.

30. Galluzzi L, Vacchelli E, Michels J, Garcia P, Kepp O, Senovilla L, Vitale I, Kroemer G (2013) Effects of vitamin B6 metabolism on oncogenesis, tumor progression and therapeutic responses. Oncogene 32, 4995-5004.

31. Zhou CQ, Yang X, Yang YP, Wan JC, Zhao CY, Peng GG (2011) Common differentially expressed proteins in substantia nigra of mouse models with chronic Parkinson's disease induced by rotenone and MPTP. J Third Mil Med Univ 33, 1432-1436.

32. Rodríguez F, Bustos MA, Zanetti MN, Ruete MC, Mayorga LS, Tomes CN (2011) $\alpha$-SNAP prevents docking of the acrosome during sperm exocytosis because it sequesters monomeric syntaxin. PLoS One 6, e21925.

33. Burgalossi A, Jung S, Meyer G, Jockusch WJ, Jahn $\mathrm{O}$, Taschenberger H, O'Connor VM, Nishiki T, et al (2010) SNARE protein recycling by $\alpha$-SNAP and $\beta$ SNAP supports synaptic vesicle priming. Neuron 68 , 473-487.

34. Barnard RJ, Morgan A, Burgoyne RD (1997) Stimulation of NSF ATPase activity by $\alpha$-SNAP is required for 
SNARE complex disassembly and exocytosis. $J$ Cell Biol 139, 875-883.

35. Johannessen M, Walquist M, Gerits N, Dragset M, Spang A, Moens U (2011) BKV agnoprotein interacts with $\alpha$-soluble $\mathrm{N}$-ethylmaleimide-sensitive fusion attachment protein, and negatively influences transport of VSVG-EGFP. PLoS One 6, e24489.

36. Xi Z, Deng W, Wang L, Xiao F, Li J, Wang Z, Wang X, Mi X, et al (2015) Association of $\alpha$-soluble NSF attachment protein with epileptic seizure. $J \mathrm{Mol} \mathrm{Neu-}$ rosci 57, 417-425.

37. Saw NM, Kang SY, Parsaud L, Han GA, Jiang T, Grzegorczyk K, Surkont M, Sun-Wada GH, et al (2011) Vacuolar $\mathrm{H}(+)$-ATPase subunits Voa1 and Voa2 cooperatively regulate secretory vesicle acidification, transmitter uptake, and storage. Mol Biol Cell 22, 3394-3409.

38. Matveeva EA, Whiteheart SW, Vanaman TC, Slevin JT (2001) Phosphorylation of the Nethylmaleimidesensitive factor is associated with depolarization dependent neurotransmitter release from synaptosomes. J Biol Chem 276, 12174-12181.

39. Napolitano A, Manini P, d'Ischia M (2011) Oxidation chemistry of catecholamines and neuronal degeneration: an update. Curr Med Chem 18, 1832-1845.

40. Jeon S, Kim YJ, Kim ST, Moon W, Chae Y, Kang M, Chung MY, Lee H, et al (2008) Proteomic analysis of the neuroprotective mechanisms of acupuncture treatment in a Parkinson's disease mouse model. Proteomics 8, 4822-4832. 